\title{
Autographa nigrisigna looper (Lepidoptera: Noctuidae) excludes parasitoid egg using cuticular encystment induced by parasitoid ovarian fluid
}

\author{
Osamu Namba, * Yutaka Nakamatsu, Ken MiUra and Toshiharu TanaKa \\ Laboratory of Applied Entomology, Graduate School of Bio-Agricultural Sciences, Nagoya University; Nagoya, Aichi 464-8601, \\ Japan
}

(Received 2 December 2007; Accepted 26 February 2008)

\begin{abstract}
Autographa nigrisigna (Lepidoptera: Noctuidae) loopers form cuticular cysts on the dorsal site of the penultimate segment by parasitization of endoparasitoid Campoletis chlorideae (Hymenoptera: Ichneumonidae) and interrupt successful parasitization by excluding the parasitoid egg and larva in the hemocoel into the cyst. This study elucidated for the first time the difference in the exclusion rates with host age at the time of parasitization. All parasitized host loopers of D1L1 (Day 1, 1st instars) and D0L2 formed cuticular cysts and nearly 70\% could exclude parasitoid eggs successfully; however, the number of loopers that could form cuticular cysts decreased with host aging at the time of parasitization. To elucidate the difference between the encapsulation system and cuticular encystment, it was examined whether the substances encapsulated are excluded by cuticular encystment. A small piece of antenna the same size as an egg used as a biotic foreign substance was absolutely encapsulated and was not excluded by encystment. Further, it was clarified whether parasitoid eggs with a different surface were encapsulated or excluded by cuticular encystment with or without ovarian fluid. UV eggs (with an intact surface and development ceased by UV treatment) were hard to encapsulate, but were excluded with encystment. Plasma eggs with a degenerate surface (treated with heating for $5 \mathrm{~min}$ at $60^{\circ} \mathrm{C}$ after 10 -min immersion in plasma of Pseudaletia separata) were encapsulated and not excluded by cuticular encystment, although ovarian fluid increased the cyst formation rate. These results show that cuticular encystment functions to exclude parasitoid eggs incapable of encapsulation.
\end{abstract}

Key words: Cuticular encystment; encapsulation; Plusiinae; looper

\section{INTRODUCTION}

It is an important for living organisms to clear foreign organisms from inside the body. Lepidopteran larvae can exclude various organisms such fungi, bacteria or parasites that invade their hemocoel by means of phagocytosis, nodule formation or encapsulation as a defence system (Vinson, 1977; Gupta, 1989; Engström, 1992; Pathak, 1993; Pech and Strand, 1995; Ribeiro et al., 1996). Larger organisms than hemocytes invading the hemocoel are particularly excluded by a cellular defense system enclosing the hemocytes, like encapsulation (Salt, 1970).

Cuticular encystment is considered another type of defense system to clear away foreign substances such as endoparasitoid eggs from the hemocoel.
Arthur and Ewen (1975) reported that Trichoplusia ni (Hübner) looper formed an epicuticular cyst on the dorsum part of the penultimate segment when it was parasitized by a solitary endoparasitoid, Banchus flavescens Cresson. Cuticular encystment has been reported in eight species of Plusiinae loopers [T. ni (Hübner) (Arthur and Ewen, 1975), Anagrapha falcifera (Kirby) and Autographa californica (Speyer) (Ewen and Arthur, 1976), A. nigrisigna Walker, Anadevidia peponis (Fabricius), T. intermixta (Warren), Acanthoplusia agnata (Staudinger) and Erythroplusia rutilifrons (Walker) (Namba et al., 2004)], and appears to be common in Plusiinae species; however, the formation rate of the cuticular cyst varied according to host stages at the time of parasitization and the host species (Namba et al., 2004). There is no information

\footnotetext{
* To whom correspondence should be addressed at: E-mail: namba.osamu@c.mbox.nagoya-u.ac.jp
} DOI: 10.1303/aez.2008.359 
about the efficacy of cuticular cyst formation in host developmental stages. It is well known that large foreign substances invading the hemocoel are encapsulated, but the question has arisen whether materials encapsulated in the hemocoel are excluded by cuticular encystment.

In this study, we firstly elucidated the difference in the exclusion rates with host age at the time of parasitization, and clarified the relationship between hemocyte response, such as encapsulation and exclusion by cuticular encystment, by using eggs with a different surface. Further, we examined the parasitoid factor causing cuticular encystment, such as an acid gland (venom), suggested by Ewen and Arthur (1976), in T. ni and B. flavescens association.

\section{MATERIALS AND METHODS}

Insect culture. Host looper A. nigrisigna were collected from cabbage fields in Nagakute, Aichi Prefecture in August 2004; however, some individuals were infected with Nosema sp. (Microsporididea). To establish a Nosema-free strain, Nosema infection was examined from each of the adult moths after egg collection. Eggs were collected from each paired moth in a vinyl bag $(23 \times 13 \mathrm{~cm})$ with a cotton pad soaked in 2-3\% sugar solution. Collected eggs were washed with $4-6 \%$ sodium hypochlorite according to Guy et al. (1985). Nosema infection was confirmed with the conventional method, that is, each whole body of the mother moth was crushed using a small clean mortar, and the smear was examined with Giemsa stain under a microscope (Sharan et al., 1992). Eggs oviposited by adult moths infected with Nosema were discarded. A selected Nosema-free strain was used as the host for experiments. Each parasitized or injected looper was reared individually on the artificial diet described previously (Kaneko, 1993; Namba et al., 2004) in small plastic Petri dishes ( $6 \mathrm{~cm}$ diameter, $1.5 \mathrm{~cm}$ high) under a long-day photoregime $(16 \mathrm{~L}: 8 \mathrm{D})$ at $25 \pm 1{ }^{\circ} \mathrm{C}$. Campoletis chlorideae (Uchida) (Hymenoptera: Ichneumonidae) is a solitary coinobiont endoparasitoid of many noctuid species (Lepidoptera: Noctuidae), including Spodoptera litura (Fabricius), Helicoverpa armigera (Hübner), Helicoverpa assulta Guenee, Pseudaletia separata (Walker), Spodoptera exigua (Hübner), Agrotis ypsilon (Rottemberg), Anomis flava (Fabricius) and Leucania loreyi Duponchel (Sato, 1988; Yan and Wang, 2006). C. chlorideae was obtained from the host larvae, S. litura, H. assulta, A. peponis and A. nigrisigna collected in cabbage fields in Nagakute from September to November, 2003. The parasitoid colony was maintained in our laboratory by using 2 nd instars of $P$. separata as a host. $C$. chlorideae parasitize younger hosts of $P$. separata successfully (Yan and Wang, 2006). After parasitization, P. separata larvae are reared on an artificial diet (Insecta ${ }^{\circledR} \mathrm{LF}$, Nihon Nohsan Co) under the same conditions as mentioned above. Newly emerged parasitoid wasps were kept in a plastic box $(30 \times 22 \times 6 \mathrm{~cm})$ for 3 days to ensure mating. Each plastic box was provided with a cotton pad soaked in 20-30\% honey solution as food. Copulated adult wasps were maintained in a plastic tube $(2.5 \mathrm{~cm}$ diameter, $9.5 \mathrm{~cm}$ long) with food until use.

Cuticular cyst formation rates of $\boldsymbol{A}$. nigrisigna loopers parasitized at various different stages. Autographa nigrisigna loopers can pupate successfully by exclusion of parasitoid eggs. As it rarely happened that a parasitoid egg hatched after being confined in a cuticular cyst, it was regarded as exclusion of the egg. To clarify the relationship between cuticular cyst formation and successful parasitization, host loopers on different developmental stages were attacked by a $C$. chlorideae female 5-14 days post-emergence and the rates of cyst formation was examined. All host loopers attacked were reared individually to check for cuticular cyst formation until pupation or parasitoid emergence every day under a dissecting microscope. A cuticular cyst formed on the dorsal side of the penultimate segment is confirmed easily as exterior swelling under a dissecting microscope with low magnification.

Formation of a cuticular cyst on looper Autographa nigrisigna responding to various stimulations. In A. nigrisigna-C. chlorideae association, it is unknown which factors are effective for forming a cuticular cyst. To clarify the stimulation factor forming a cuticular cyst, four components, that is eggs, polydnavirus (PDV), ovarian fluid (OVF) and venom were taken from ovaries, and artificially injected into the looper. C. chloridae females have a typical ovary with a calyx region that produces PDV (Tian et al., 2007) and a poorly developed venom gland and reservoir like C. sonorensis (Nor- 
ton et al., 1975; Webb and Strand, 2005). Lateral oviducts are filled with eggs, OVF and PDV. Ovaries were pulled out of the abdomen by clipping the ovipositor gently with fine forceps in physiological saline (PBS: $0.87 \% \mathrm{NaCl}$ in $0.067 \mathrm{M}$ phosphate buffer, $\mathrm{pH}$ 7.4). Venom reservoirs with filaments were separated from ovaries and placed in a dimple dish. Venom fluid was collected after spinning at $15,000 \mathrm{rpm}$ for $10 \mathrm{~min}$. Lateral oviducts after removing Dufour's gland were torn with fine forceps and spun for $10 \mathrm{~min}$ at $2,000 \mathrm{rpm}$ to remove the cell debris and eggs. The resulting supernatant was centrifuged at $15,000 \mathrm{rpm}$ for $10 \mathrm{~min}$ to separate PDV from OVF. The supernatant was adjusted to one female equivalent per microliter with PBS and used as ovarian fluid. The precipitate was used as PDV by adjusting to one female equivalent per microliter with PBS. Matured eggs were collected from lateral oviducts. Activated eggs (i.e., within 3 hours after oviposition) were retrieved from parasitized loopers. Both mature and activated eggs were centrifuged at $2,000 \mathrm{rpm}$ for $10 \mathrm{~min}$, and rinsed three times with PBS.

D1L2 and D0L3 loopers were used for material injection, because younger loopers were too small to inject materials artificially. Fourth and 5th instars were not suitable for this study judging from the results of parasitization data (Fig. 1). Each of three components (OVF, PDV and venom) was injected through a proleg of nonparasitized larva after narcotizing with $\mathrm{CO}_{2}$ using a fine glass needle. Eggs were singly injected through a proleg in D0L3 loopers. D1L2 loopers were injected through a cervical location between the head and thorax because younger loopers before D1L2 were too small to inject eggs through a proleg successfully. The height $(\mu \mathrm{m})$ of the cyst was measured 3-4 days after parasitoid attack when the cuticular cyst reached the maximum size.

Morphological observation of cuticular cyst. To clarify the morphological difference in host loopers injected with various stimulants, 5 or 6 loopers in each case were fixed with $2.5 \%$ glutaraldehyde solution, post-fixed with Bouin's solution for $24 \mathrm{~h}$, dehydrated and embedded in paraffin. Eight-micrometer sections were stained with Mayer's hematoxylin and 1\% eosin Y solution (Sano, 1965). Further to examine amorphous materials secreted in a cyst, paraffin sections $(20 \mu \mathrm{m}$ thick) were double-stained with two kinds of lectins, rhodamine succinylated wheat germ agglutinin (Rhodamin-s-WGA, Vector Lab., USA, $10-20 \mu \mathrm{g} / \mathrm{ml}$ in PBS) and fluorescein tomato lectin (Fluorescein-LEL, Vector Lab. adjusted to the same concentration) for $2 \mathrm{~h}$, then rinsed with PBS three times before observation using a confocal laser scanning microscope (LSM5 Pascal, Zeiss, Germany). WGA and LEL are lectins with binding sites specific for $\mathrm{N}$-acetylglucosamine residues (Goldstein et al., 1975; Ward et al., 1985; Greven and Peters, 1986).

Response of loopers to artificially injected eggs with different surfaces. To examine the response of loopers to foreign substances, eggs after various treatments were injected into unparasitized and parasitized hosts and were observed as to whether they were encapsulated or excluded by cuticular encystment. To make eggs that ceased development but still possessed an intact surface, the activated eggs above were treated with ultraviolet (UV) light at $5 \mathrm{~cm}$ distance for $3 \mathrm{~min}$, and were used as UV eggs. One percent SDS was reported to be effective to modify the parasitoid egg surface (Asgari and Schmidt, 1994). UV eggs were immersed in $1 \%$ SDS-PBS for $5 \mathrm{~min}$; however, SDS eggs were not encapsulated in the host at least $24 \mathrm{~h}$ after injection $(n=12$, singly injected in a cervical place of D0L3). Heat treatment of eggs also was not effective to alternate the egg surface, because heating at $98^{\circ} \mathrm{C}$ for $5 \mathrm{~min}$ gave almost the same result as UV treatment alone (no encapsulation $62.5 \%$, exclusion $37.5 \%, N=8$ ). Further, to make eggs with a degenerated surface, UV eggs were first immersed in plasma of $P$. separata for $10 \mathrm{~min}$ at $25^{\circ} \mathrm{C}$, following by heating for $5 \mathrm{~min}$ at $60^{\circ} \mathrm{C}$, and used as plasma eggs. Cell-free hemolymph, as plasma, was adjusted as follows: hemolymph from day 3-4 last instars of $P$. separata after narcotization was collected on ice, $8 \%$ saturated 1-phenyl-2thiourea (PTU) per microliter hemolymph in volume was immediately added, and then centrifuged to remove hemocytes at $2,000 \mathrm{rpm}$ for $10 \mathrm{~min}$ at $4{ }^{\circ} \mathrm{C}$. Treated eggs were injected into a cervical location between the head and thorax with a fine glass needle-like injection of matured and activated eggs. A small piece of antenna was used as biotic foreign material. A piece of antenna from a $C$. chlorideae female was cut to the same size as the parasitoid egg and heated for $5 \mathrm{~min}$ at $60^{\circ} \mathrm{C}$ before injection. The result (Table 1) that ovarian fluid 
was most effective for cuticular cyst formation among the three components (OVF, PDV and venom) led us to confirm whether the encapsulation rate was enhanced instead of forming a cuticular cyst when no cyst was formed or whether another stimulant formed a cuticular cyst. With a dual injection, only one egg was injected per looper within a few minutes following injection with OVF (3-4/10 female equivalent per looper). Loopers were dissected in PTU-PBS ( $8 \%$ to saturated PTU in PBS) 3 days after injection to observe the encapsulation degree after confirming cuticular cyst formation under a dissecting microscope.

\section{RESULTS}

Cuticular cyst formation rates on $A$. nigrisigna loopers parasitized at various different stages

In early stages, such as D1L1 and D0L2, all loopers formed cuticular cysts (CF) and nearly $70 \%$ host loopers could pupate by exclusion of parasitoid egg or larva, but about $20 \%$ host loopers were killed successfully by parasitoid larvae egression (Fig. 1). Data were divided between loopers forming a cuticular cyst (CF) and those that failed to form a cuticular cyst (NCF) in the developmental stage of the host looper at parasitization. The pupation rate of CF loopers decreased with the age of host loopers at the time of parasitization, while the number of NCF loopers increased gradually in 2nd and later instars (Fig. 1). Almost all D1L2 and D0L3 loopers in NCF were killed by successful parasitism. About half of all D0L4 loopers could form cysts (CF) but half failed to do so (NCF). More than $80 \%$ of parasitized D1L4 loopers could not form a cuticular cyst (NCF) and low successful parasitization was observed (Fig. 1). These results mean that $C$. chlorideae failed to parasitize older host loopers like late 4 th instars.

Formation of cuticular cyst on looper Autographa nigrisigna responding to various stimuli

Three components (OVF, PDV and venom) injected with the egg at oviposition, were examined to clarify the stimulation factor to form a cuticular cyst. OVF showed the strongest stimulant action to form a cuticular cyst among substances. The rate of cuticular cyst formation was $77.8 \%$ and $66.7 \%$ in D1L2 and D0L3, respectively (Table 1). Venom or PDV from C. chlorideae did not form a cyst on

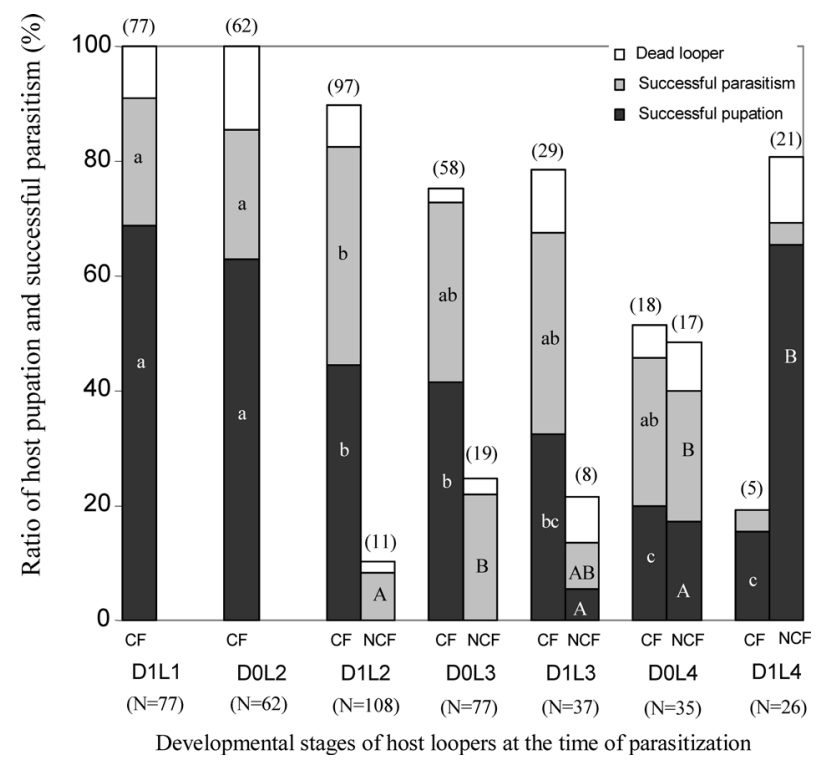

Fig. 1. Ratio of cuticular cyst formation on A. nigrisigna loopers attacked at various different stages. CF: loopers formed a cuticular cyst, NCF: loopers failed to form a cuticular cyst. Stage of each looper attacked by $C$. chloridae is shown as day-instar (i.e. D1L1: day 1, 1st instar). Different letters in the same pattern of the same column (CF or NCF) indicate significant difference by chi-square independent test $(p<0.05)$ for respective values between two after one-way ANOVA.

loopers, similar to saline. A female wasp injects an egg with OVF, venom and PDV in the host at oviposition. Furthermore, we paid attention to the potential of eggs as a stimulant to form a cuticular cyst. Cyst formation in parasitized hosts as a positive control was about $80 \%$ in both D1L2 and D0L3. Injection of only matured and activated eggs showed that eggs worked as a stimulant in cyst formation, although they had a little less stimulant action than OVF (Table 1); however, cuticular cysts were similar in size to those in parasitized loopers.

The OVF dose response was acquired in D1L2 loopers (Fig. 2). One tenth-diluted OVF gave a $70 \%$ response in forming a cyst. Parasitization caused about $80 \%$ cyst formation in D1L2 and showed a similar rate to that of a one-tenth dose. Forty to 160 times dilution of one female equivalent gave about $20 \%$ cyst formation (Fig. 2).

\section{Morphological observation of cuticular cyst}

Epidermal cells became thickened and secreted cuticular-like material (clm) into the cyst space with OVF stimulation (Fig. 3A), contrary to no 
Table 1. Rate of cuticular cyst formation on looper Autographa nigrisigna to various stimulation

\begin{tabular}{|c|c|c|c|c|}
\hline \multirow{3}{*}{$\begin{array}{c}\text { Materials } \\
\text { injected into } \\
\text { unparasitized } \\
\text { hosts }\end{array}$} & \multicolumn{4}{|c|}{ Developmental stage of loopers injected } \\
\hline & \multicolumn{2}{|c|}{$\mathrm{D} 1 \mathrm{~L} 2^{\mathrm{a}}$} & \multicolumn{2}{|c|}{ D0L $3^{\mathrm{a}}$} \\
\hline & $\begin{array}{l}\text { Cyst formation rate } \\
\qquad(\%)\end{array}$ & $\begin{array}{l}\text { Average height of cyst } \\
\qquad(\mu \mathrm{m})\end{array}$ & $\begin{array}{l}\text { Cyst formation rate } \\
\qquad(\%)\end{array}$ & $\begin{array}{l}\text { Average height of cyst } \\
\qquad(\mu \mathrm{m})\end{array}$ \\
\hline Ovarian fluid & $77.8\left(28^{b} / 36\right) a^{c}$ & $242 \pm 33 \mathrm{a}^{\mathrm{d}}$ & $66.7\left(18^{b} / 27\right) a^{c}$ & $256 \pm 67 \mathrm{a}^{\mathrm{d}}$ \\
\hline Polydnavirus & $4.5(2 / 44) b$ & - & $6.7(1 / 15) b$ & - \\
\hline Venom & $2.2(1 / 45) b$ & - & $0(0 / 15)$ & - \\
\hline PBS & $7.1(3 / 42) b$ & - & $6.7(1 / 15) b$ & - \\
\hline Matured eggs ${ }^{\mathrm{e}}$ & $69.2(9 / 13) \mathrm{a}$ & $240 \pm 89 a$ & $50.0(10 / 20) \mathrm{a}$ & $198 \pm 49 a$ \\
\hline Activated eggs ${ }^{\mathrm{f}}$ & $73.5(25 / 34) \mathrm{a}$ & $203 \pm 58 \mathrm{a}$ & $58.3(42 / 72) \mathrm{a}$ & $199 \pm 70 \mathrm{a}$ \\
\hline Parasitized host & $80.0(40 / 45) \mathrm{a}$ & $221 \pm 72 \mathrm{a}$ & $80.8(21 / 26) \mathrm{a}$ & $203 \pm 62 a$ \\
\hline
\end{tabular}

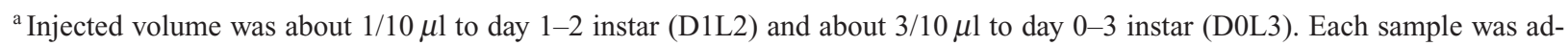
justed to one female equivalent per one $\mu$ l.

${ }^{\mathrm{b}}$ Number of loopers that formed cuticular cysts in hosts injected with each material.

${ }^{c}$ Different letters in the same column indicate statistical difference by contingency chi-square analysis $(p<0.01)$ for respective values except less than 5, by Fisher's exact test.

${ }^{\mathrm{d}}$ Same letter in the same column indicates non-significant difference by one-way ANOVA $(p<0.01)$.

${ }^{\mathrm{e}}$ Mature eggs were taken from lateral oviducts of Campoletis chlorideae.

${ }^{\mathrm{f}}$ Activated eggs were taken from parasitized loopers within $3 \mathrm{~h}$ after oviposition. One egg was injected per a looper.

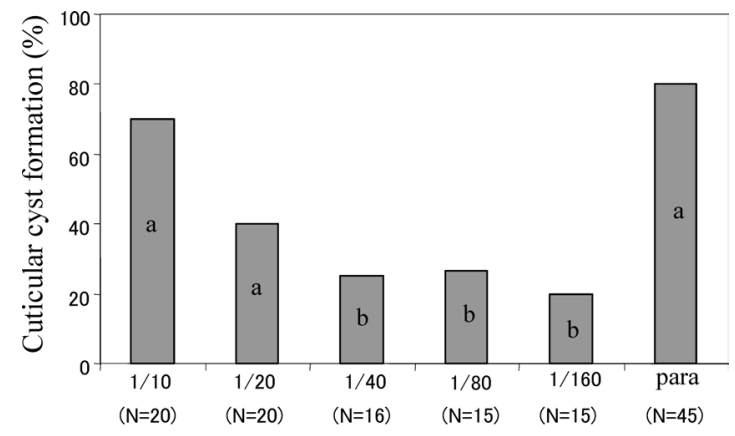

Female equivalent of ovarian fluid

Fig. 2. Dose responses of A. nigrisigna looper to various concentrations of ovarian fluid from $C$. chlorideae. Injection was performed on day 1, 2nd instar loopers. Rate of cyst formation was examined day 3 after injection. $\mathrm{N}$ indicates the number of loopers injected with each concentration of ovarian fluid. Para: parasitized control. Different letters in each bar indicate significant difference by one-way ANOVA $(p<0.05)$.

thickening of the epidermis in control samples (Fig. 3B). OVF stimulation increased the height of epidermal cells compared to those with normal parasitization (Fig. 3C). UV eggs also stimulated epidermal cells to secrete clm $72 \mathrm{~h}$ after injection (Fig. 3D). The thickened epidermal layer secreted cuticular-like material stained with fluorescein tomato lectin (LEL) in the inter-space of cysts (Fig. $3 \mathrm{E})$.

\section{Response of host loopers to injected materials by encapsulation or cuticular encystment}

To clarify the relationship between the encapsulation reaction and cuticular encystment, the response to parasitoid eggs was examined. UV eggs with an intact egg surface could avoid a host encapsulation reaction and about $30 \%$ UV eggs were excluded by cuticular encystment (Table 2). Plasma eggs with a degenerated surface were encapsulated at a rate of $40-60 \%$ regardless of OVF coexistence, but no egg was excluded by encystment. An antenna used as a positive control was absolutely encapsulated and was not excluded by encystment.

To examine the response against eggs with a different surface in the hemolymph of hosts regulated under normal parasitization, the same materials were injected into hosts within $10 \mathrm{~min}$ after parasitization. UV eggs and plasma eggs were easy to distinguish from normal eggs oviposited by females because of the different egg surface. More than one-half of the parasitized hosts formed cuticular cysts and excluded $13 \%$ of UV eggs and $15 \%$ 
O. NAMBA et al.

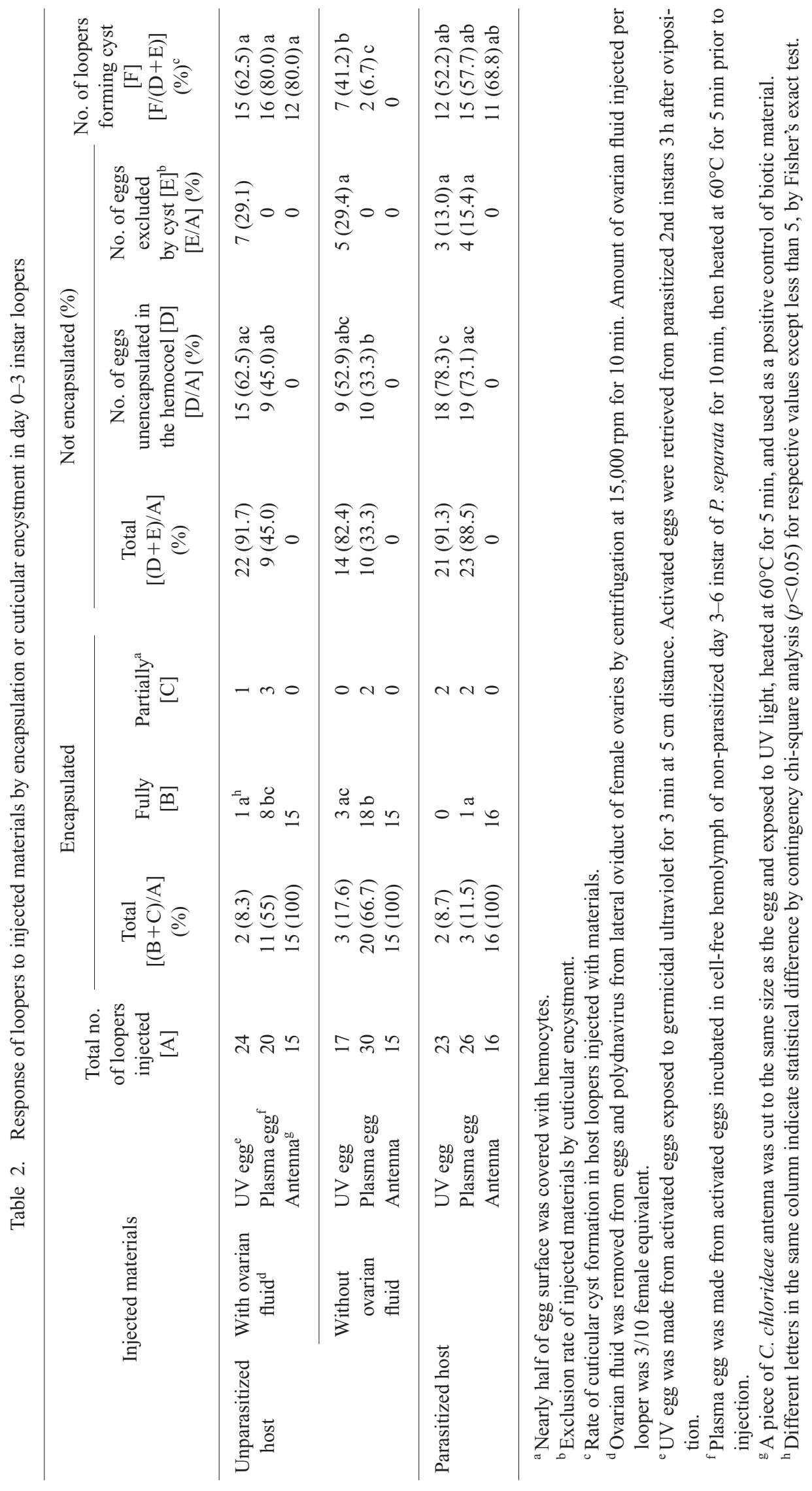



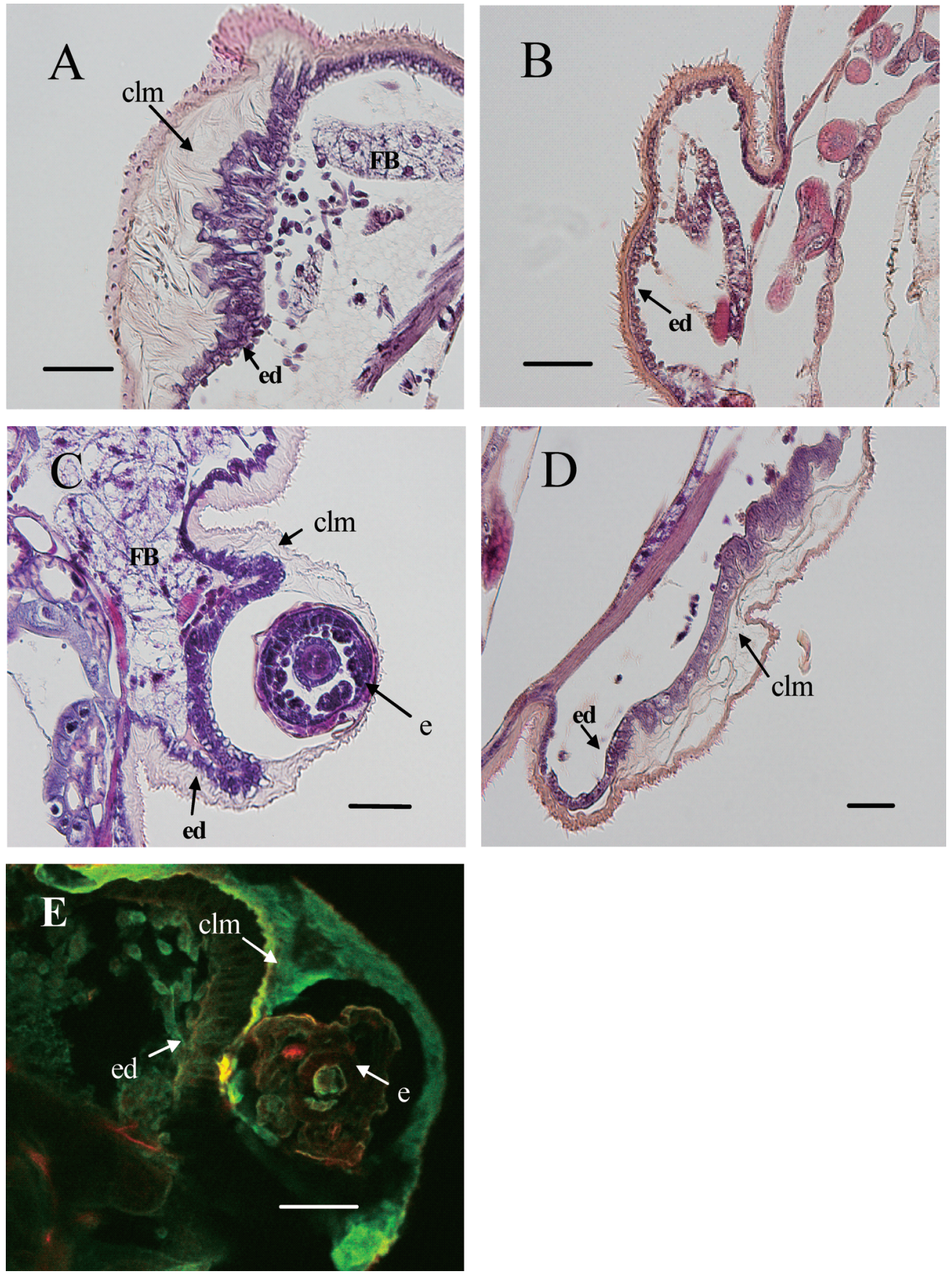

Fig. 3. Morphological change of epidermis on penultimate segment by injection of various materials. A: Host epidermal cells were thickened and secreted cuticular-like materials (clm) in inter-space of cyst $72 \mathrm{~h}$ after ovarian fluid (OVF) injection. B: Control of epidermal cells at the same developmental stage. C: Cuticular cyst of host looper $39 \mathrm{~h}$ after oviposition. Campoletis chlorideae egg (e) is excluded in the cuticular cyst. Epidermal cells are thickened and secret clm. D: Injection of UV eggs also resulted in thickened epidermal cells and clm secretion $72 \mathrm{~h}$ after injection. E: Space in cuticular cyst was filled with clm stained with fluorescein tomato lectin (LEL) $72 \mathrm{~h}$ after oviposition. e: parasitoid egg. FB: fat body, ed: epidermis. Bar $=50 \mu \mathrm{m}$.

of plasma eggs by encystment without encapsulation.

\section{DISCUSSION}

Although it was reported that cuticular encystment worked as an exclusion mechanism in Plusi- 
inae loopers (Arthur and Ewen, 1975; Ewen and Arthur, 1976; Namba et al., 2004), there was no information about the exclusion rate of parasitoid eggs with the aging of loopers at the time of parasitization. Our results show that younger loopers as late 1 st and early 2 nd instars are able to pupate by excluding parasitoid eggs during development even after parasitization; however, the pupation rate from late 2 nd to late $3 \mathrm{rd}$ instars also decreased gradually. In particular, older loopers, such as 4th instars, were unsuitable for C. chlorideae parasitization in spite of oviposition, because more than $80 \%$ of D1L4 loopers could not form a cuticular cyst and most pupated.

Ovarian fluid induced cuticular cyst formation in the penultimate segment of $A$. nigrisigna. On the other hand, venom fluid provided no stimulation to form a cuticular cyst, unlike in the $B$. flavescens- $T$. $n i$ system (Ewen and Arthur, 1976). Although the cause of this difference remains unclarified, it seems reasonable that venom function transfers to that of ovarian fluid. Both mature and activated eggs act as stimulants to form a cyst just like OVF. Mature eggs were taken from lateral oviducts filled with OVF. It is considered that washing with saline may be not enough to remove OVF components from the egg surface. Although oviposited eggs seem to have their surface altered by the action of the host hemolymph, the altered surface could still stimulate epidermal cells to form a cyst. Davies and Vinson (1986) reported that in Cardiochiles nigriceps- $H$. virescens association, mature eggs obtained from lateral oviducts or from parasitized hosts within $1 \mathrm{~h}$ after oviposition are not encapsulated in vitro, or in vivo after $24 \mathrm{~h}$, whereas all immature eggs located within the ovariole are encapsulated. A fibrous layer surrounding the egg surface seems to protect the egg from encapsulation. Asgari and Schmidt (1994) also reported a protective coat on the egg surface, which can be removed easily by SDS detergent. In the case of C. chlorideae, SDS detergent had no effect on the degeneration of the egg surface. UV eggs can also escape from encapsulation of the host and stimulate the epidermal cells to form a cyst, probably due to the same intact surface as the activated eggs. The fact that about $50 \%$ of UV eggs were not encapsulated supports the finding that half of parasitized host loopers are killed by successful parasitism. If oviposited eggs develop without artificial aborting by UV, parasitization will be successful at $50 \%$. This means that the egg surface itself may act to avoid host encapsulation rather than coating material on the egg surface. The egg surface seems to stimulate epidermal cells. Materials injected into the host hemocoel were probably carried with backward blood-flow through the body cavity until they finally reached the penultimate segment which is opened around blood vessels. It is still unknown why only epidermal cells around the penultimate segment respond to OVF stimulation or UV eggs.

Plasma eggs injected into unparasitized loopers without ovarian fluid were encapsulated at a high rate and were difficult to exclude. Plasma eggs possess a degenerated surface from the effect of host hemolymph and heating; however, in parasitized loopers, the encapsulation rate decreased to $3.8 \%$, although it became $11.5 \%$ if adding the rate of partially encapsulated eggs. Plasma eggs were hard to recognize as foreign substances in hemolymph conditioned with OVF and PDV plus venom. Recognition of foreign substances in parasitized hosts seems not to be simply explained by differences in the egg surface. The small pieces of antennae from female wasps used as biotic material were completely encapsulated as foreign substances even when the loopers were parasitized. None of the injected antenna was confined in the cyst. This shows that encapsulated foreign substances are not excluded by cuticular encystment. The encapsulated foreign substance probably did not move into the host hemocoel by attaching to some organs like the fat body or trachea.

Our results suggest that $A$. nigrisigna has evolved the cuticular encystment mechanism to exclude parasitoid eggs that are not encapsulated in the host hemocoel. This is especially true in younger instar loopers that do not develop sufficient encapsulation response to foreign substances; therefore, cuticular encystment may be effective to exclude foreign substances.

\section{ACKNOWLEDGEMENTS}

We express our thanks to M. A. M. Bautista for proofreading the manuscript and helpful comments. This work was supported in a part by a Grant-in-Aid for Scientific Research Work (houga) No. 16658024 from the Ministry of Education, Culture, Sports, Science and Technology of Japan.

\section{REFERENCES}

Arthur, A. P. and A. L. B. Ewen (1975) Cuticular encyst- 
ment: a unique and effective defense reaction by cabbage looper larvae against parasitism by Banchus flavescens (Hymenoptera: Ichneumonidae). Ann. Entomol. Soc. Am. 68: 1091-1094.

Asgari, S. and O. Schmidt (1994) Passive protection of eggs from the parasitoid Cotesia rubecula, in the host, Pieris rapae. J. Insect Physiol. 40: 789-795.

Davies, D. H. and S. B. Vinson (1986) Passive evasion by eggs of braconid parasitoid Cardiochiles nigriceps of encapsulation in vitro by haemocytes of host Heliothis virescens. Possible role for fibrous layer in immunity. $\mathrm{J}$. Insect Physiol. 32: 1003-1010.

Engström, Y. (1992) Insect immune systems. In Insect Molecular Science. 16th Symposium of the Royal Entomological Society of London, 12-13 September 1991 at Imperial College, London (J. M. Crampton and P. Eggleston, eds.). Academic Press, London, pp. 125-137.

Ewen, A. L. B. and A. P. Arthur (1976) Cuticular encystment in three noctuid species (Lepidoptera): induction by acid gland secretion from an ichneumonid parasite (Banchus flavescens). Ann. Entomol. Soc. Am. 69: 1087-1090.

Goldstein, I. J., S. Hammarström and G. Sundblad (1975) Precipitation and carbohydrate-binding specificity studies on wheat germ agglutinin. Biochim. Biophy. Acta 405: 53-61.

Greven, H. and W. Peters (1986) Localization of chitin in the cuticle of Tardigrada using wheat germ agglutinin-gold conjugate as a specific electron-dense marker. Tissue Cell 18: 297-304.

Gupta, A. P. (1989) Insect host immune system and endocytobionts: their avoidance strategies. In Insect Endocytobiosis. Morphology, Physiology, Genetics, Evolution (W. Schwemmlar, ed.). CRC Press, Florida, pp. 11-20.

Guy, R. H., N. C. Leppla, J. R. Rye, C. W. Green, S. L. Barrette and K. A. Hollien (1985) Trichoplusia ni. In Handbook of Insect Rearing. Vol. 2 (P. Singh and R. F. Moore, eds.). Elsevier Science Publishers, Amsterdam, pp. $487-494$.

Kaneko, J. (1993) Existence ratio of silvery moth, Autographa nigrisigna (L.) after overwintering in cabbage field at Sapporo. Ann. Rep. Soc. Plant Prot. North Japan 44: 124-126 (in Japanese).

Namba, O., M. Saigusa and T. Tanaka (2004) Cuticular encystment in larvae of Plusiinae looper (Lepidoptera: Noctuidae) parasitized by the gregarious endoparasitoid Cotesia glomerata. Ann. Entomol. Soc. Am. 97: 976-981.

Norton, W. N., S. B. Vinson and D. B. Stoltz (1975) Nuclear secretory particles associated with the calyx cells of the ichneumonid parasitoid Campoletis sonorensis (Cameron). Cell Tissue Res. 162: 195-208.

Pathak, J. P. N. (1993) Cell mediated defense reactions in insects. In Insect Immunity (J. P. N. Pathak, ed.). Kluwer Academic Publishers, London, pp. 47-58.

Pech, L. L. and M. R. Strand (1995) Encapsulation of foreign targets by hemocytes of the moth Pseudoplusia includens (Lepidoptera: Noctuidae) involves an RGD-dependent cell adhesion mechanism. J. Insect Physiol. 41: $481-488$.

Ribeiro, C., N. Simoes and M. Brehelin (1996) Insect immunity: the haemocytes of the armyworm Mythimna unipuncta (Lepidoptera Noctuidae) and their role in defense reactions. In vitro and in vivo studies. J. Insect Physiol. 42: 815-822.

Salt, G. (1970) The Cellular Defense Reactions of Insects. Cambridge Monographs in Experimental Biology, 16. Cambridge Univ. Press, Cambridge, UK. pp. 118.

Sano, Y. (1965) Histological Techniques. Nanzando Company, Tokyo, pp. 143-206 (in Japanese).

Sato, Y. (1988) The World of Parasitic Wasps. Tokai University Press, Kanagawa, Japan. pp. 242 (in Japanese).

Sharan, S. K., A. K. Bansal, R. M. Shukla and K. Thangavelu (1992) A new method of detection of pebrine disease in tasar silk moth, Antheraea mylitta Drury (Saturniidae). J. Res. Lepidoptera 31(1-2): 12-15.

Tian, S. P., J. H. Zhang and C. Z. Wang (2007) Cloning and characterization of two Campoletis chlorideae ichnovirus vankyrin genes expressed in parasitized host Helicoverpa armigera. J. Insect Physiol. 53: 699-707.

Vinson, S. B. (1977) Insect host responses against parasitoids and the parasitoid's resistance: with emphasis on the Lepidoptera-Hymenoptera association. In Comparative Pathology. Vol. 3 (L. A. Bulla, Jr. and T. C. Cheng, eds.). Plenum, New York, pp. 103-125.

Ward, H. D., J. Alroy, B. I. Lev, G. T. Keusch and M. E. Pereira (1985) Identification of chitin as a structural component of giardia cysts. Infect. Immun. 49: 629-634.

Webb, B. A. and M. R. Strand (2005) The biology and genomics of polydnaviruses. In Comprehensive Molecular Insect Science. Vol. 6 (L. I. Gilbert, K. Iatrou and S. S. Gill, eds.). Elsevier, San Diego, pp. 323-360.

Yan, Z.-G. and C.-Z. Wang (2006) Identification of Mythmna separata-induced maize volatile synomones that attract the parasitoid Campoletis chlorideae. J. Appl. Entomol. 130: 213-219. 